\title{
Thermodynamic analysis of variable viscosity MHD unsteady generalized Couette flow with permeable walls
}

\author{
David Theuri $^{1}$, Oluwole Daniel Makinde ${ }^{2}$ \\ ${ }^{1}$ Department of Pure and Applied Mathematics, Jomo Kenyatta University of Agriculture and Technology, Nairobi, Kenya \\ ${ }^{2}$ Faculty of Military Science, Stellenbosch University, Private Bag X2, Saldanha 7395, South Africa
}

Email address:

dtheuri@gmail.com (D. theuri), makinded @gmail.com (O. D. Makinde)

\section{To cite this article:}

David Theuri, Oluwole Daniel Makinde. Thermodynamic Analysis of Variable Viscosity MHD Unsteady Generalized Couette Flow with Permeable Walls. Applied and Computational Mathematics. Vol. 3, No. 1, 2014, pp. 1-8. doi: 10.11648/j.acm.20140301.11

\begin{abstract}
The thermodynamic first and second law analyses of a temperature dependent viscosity hydromagnetic generalized unsteady Couette flow with permeable walls is investigated. The transient model problem for momentum and energy balance is tackled numerically using a semi-discretization method while the steady state boundary value problem is solved by shooting method together with Runge-Kutta-Fehlberg integration scheme. The velocity and the temperature profiles are obtained and are utilized to compute the skin friction coefficient, Nusselt number, entropy generation rate and the Bejan number. Pertinent results are presented graphically and discussed quantitatively.
\end{abstract}

Keywords: Unsteady Couette Flow, Magnetic Field, Variable Viscosity, Permeable Walls, Heat Transfer, Entropy Generation, Bejan Number

\section{Introduction}

Thermodynamic irreversibility in the flow system provides information on the energy and power losses in the system. Minimization of entropy generation in the flow system enables for the parametric optimization of the system operation [1]. Thermodynamics analysis has three worthy foundations which basically are described by mass conservation equation, and the first and second law of thermodynamics [2]. The conservation of mass law explains the interactions of mass inside of a system and the first law clarifies the exchanges of energy from one system form to another system, and energy conservation in total. The second law gives a quantitative relation of the irreversibility of a system through entropy generation, the greater degree of irreversibility, the bigger of entropy production and the more energy consumption. Entropy generation destroy the available energy in the system. Therefore, in industrial and engineering systems such as magnetohydrodynamic (MHD) micro-pumps, microelectronic devices, electronic packages, cooling of nuclear reactors, MHD marine propulsion, MHD lubrication, etc., entropy generation minimization technique is important to optimize the performance of thermal-fluid devises. A magnetohydrodynamic (MHD) phenomenon refers to the outcome of mutual interaction between magnetic field and electrically conducting fluid flowing across it. Since the pioneering work of Bejan [3,4] on entropy generation minimization on engineering systems, considerable research studies have been carried out to examine entropy generation in fluid flows for various applications [5]. Salas et al. [6] analysed the second law for MHD induction devices, such as electromagnetic pumps, and electrical generators. Mahmud and Fraser [7] analytically investigated the entropy generation due to free convection in a porous cavity in the presence of magnetic field. Ibanez and Cuevas [8] considered a stationary buoyant MHD flow of a liquid metal immersed in a MHD flow through a vertical rectangular duct. They obtained the optimum conductance ratio of the wall in which the entropy generation is minimized. Eegunjobi and Makinde [9] studied the effect of Navier slip on entropy generation in a porous channel with suction/injection. The effect of Newtonian heating on entropy generation rate in a channel with permeable walls was reported by Makinde and Eegunjobi [10]. From the literature survey, it seems that the problem of entropy generation in a variable viscosity generalized unsteady MHD Couette flow with permeable boundaries has not received much attention.

In this present study, both first and second laws of thermodynamics are employed to analyse the unsteady MHD Couette flow with variable viscosity and 
suction/injection. The transient problem is tackled numerically using a semi-discretization finite difference method while shooting method coupled with a RungeKutta-Fehlberg integration scheme is employed to solve the steady state problem. Pertinent results are presented graphically and discussed quantitatively.

\section{Problem Formulation}

We consider an unsteady, incompressible, laminar flow of an electrically conducting variable viscosity fluid between a fixed permeable lower plate and a moving permeable upper plate. The fluid is acted upon by a constant pressure gradient and an external uniform magnetic field is applied perpendicular to the plates as illustrated in figure 1. It is assumed that the fluid is injected uniformly into the channel at the lower plate while the uniform fluid suction occurs at the moving upper plate. A transverse magnetic field with strength $B_{0}$ is applied parallel to the $y$-axis. There is no applied voltage and the magnetic Reynolds number is small, hence the induced magnetic field and Hall effects are negligible.

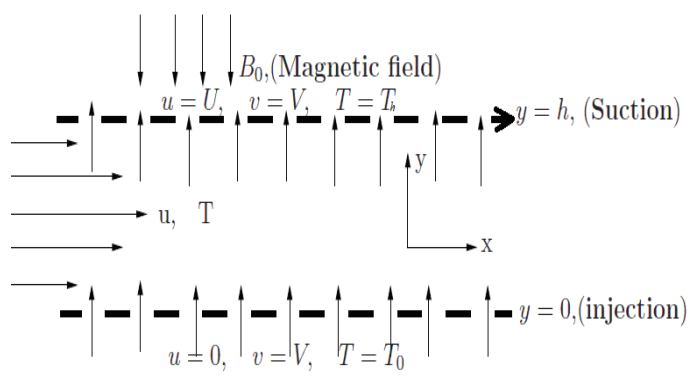

Figure 1. Schematic diagram of the problem

Under these assumptions, the governing equations for the momentum and energy balance in one dimension can be written as follows [5-10]

$$
\begin{aligned}
& \frac{\partial u}{\partial t}+V \frac{\partial u}{\partial y}=-\frac{1}{\rho} \frac{\partial P}{\partial x}+\frac{1}{\rho} \frac{\partial}{\partial y}\left(\bar{\mu}(T) \frac{\partial u}{\partial y}\right)-\frac{\sigma B_{0}^{2} u}{\rho} \\
& \frac{\partial T}{\partial t}+V \frac{\partial T}{\partial y}=\alpha \frac{\partial^{2} T}{\partial y^{2}}+\frac{\bar{\mu}(T)}{\rho c_{P}}\left(\frac{\partial u}{\partial y}\right)^{2}+\frac{\sigma B_{0}^{2} u^{2}}{\rho c_{p}}
\end{aligned}
$$

subject to the following initial and boundary conditions:

$$
\begin{gathered}
u(y, 0)=0, T(y, 0)=0 \\
u(0, t)=0, T(0, t)=T_{0} \\
u(h, t)=U, T(h, t)=T_{h}
\end{gathered}
$$

where $h$ is the channel width, $u$ is the velocity of the fluid, $t$ is the time, $P$ is the fluid pressure, $V$ is the uniform suction / injection velocity at the channel walls, $U$ is the uniform velocity of the upper wall, $\alpha$ is the thermal diffusivity, $\rho$ is the fluid density, $\sigma$ is the fluid electrical conductivity, $k$ is the thermal conductivity coefficient, $c_{p}$ is the specific heat at constant pressure, $T$ is the fluid temperature, $T_{0}$ is the lower stationary wall temperature and $T_{0}$ is the upper wall temperature. The temperature dependent viscosity $\bar{\mu}$ can be expressed as

$$
\bar{\mu}(T)=\mu_{0} e^{-m\left(T-T_{0}\right)}
$$

where $m$ is a viscosity variation parameter and $\mu_{0}$ is the fluid dynamic viscosity at lower fixed wall. Using (1) and (3b), the constant axial pressure gradient will be given as

$$
-\frac{1}{\rho} \frac{\partial p}{\partial x}=\frac{\sigma B_{0}^{2} U}{\rho}
$$

and equations (1) and (2) then become

$$
\begin{gathered}
\frac{\partial u}{\partial t}+V \frac{\partial u}{\partial y}=\frac{1}{\rho} \frac{\partial}{\partial y}\left(\bar{\mu}(T) \frac{\partial u}{\partial y}\right)-\frac{\sigma B_{0}^{2}(u-U)}{\rho} \\
\frac{\partial T}{\partial t}+V \frac{\partial T}{\partial y}=\alpha \frac{\partial^{2} T}{\partial y^{2}}+\frac{\bar{\mu}(T)}{\rho c_{P}}\left(\frac{\partial u}{\partial y}\right)^{2}+\frac{\sigma B_{0}^{2}}{\rho c_{p}}(u-U)^{2}
\end{gathered}
$$

We introduce the following non-dimensional quantities:

$$
\tau=\frac{t V}{h}, \eta=\frac{y}{h}, \alpha=\frac{k}{\rho c_{p}}, w=\frac{u}{U}, \theta=\frac{T-T_{0}}{T_{h}-T_{0}}, \bar{P}=\frac{p h}{\mu_{0} U}, \mu=\frac{\bar{\mu}}{\mu_{0}}, v=\frac{\mu_{0}}{\rho}
$$

Substituting equation (8) into equations (6)-(7), we obtain

$$
\begin{gathered}
\operatorname{Re} e^{\varepsilon \theta} \frac{\partial w}{\partial \tau}=\frac{\partial^{2} w}{\partial \eta^{2}}-\varepsilon \frac{\partial \theta}{\partial \eta} \frac{\partial w}{\partial \eta}-e^{\varepsilon \theta}\left(\operatorname{Re} \frac{\partial w}{\partial \eta}+H a(w-1)\right) \\
\operatorname{Pr} \operatorname{Re} \frac{\partial \theta}{\partial \tau}=\frac{\partial^{2} \theta}{\partial \eta^{2}}-\operatorname{Re} \operatorname{Pr} \frac{\partial \theta}{\partial \eta}+E c \operatorname{Pr} e^{-\varepsilon \theta}\left(\frac{\partial w}{\partial \eta}\right)^{2}+E c \operatorname{Pr} H a(w-1)^{2}
\end{gathered}
$$

with

$$
\left.\begin{array}{c}
w(\eta, 0)=0, \theta(\eta, 0)=0 \\
w(0, \tau)=0, \theta(0, \tau)=0 \\
w(1, \tau)=1, \theta(1, \tau)=1
\end{array}\right\}
$$

where

$\operatorname{Re}=\frac{V h}{v}$ (Reynolds number),

$\operatorname{Pr}=\frac{v}{\alpha}$ (Prandtl number),

$E c=\frac{U^{2}}{c_{p}\left(T_{h}-T_{0}\right)}($ Eckert number $)$,

$H a=\frac{\sigma B_{0}^{2} h^{2}}{\mu_{0}}$ (Magnetic field parameter),

$\varepsilon=m\left(T_{h}-T_{0}\right)$ (Viscosity exponent).

Other important physical quantities of interest in this problem are the skin friction coefficient $C_{f}$ and Nusselt number $\mathrm{Nu}$ which are defined as:

$$
C_{f}=\frac{h \tau_{w}}{\mu_{0} U}, N u=\frac{q_{w} h}{k\left(T_{h}-T_{0}\right)}
$$

where the skin friction $\tau_{w}$, heat flux $q_{w}$ at the channel walls are given by 


$$
\tau_{w}=\left.\bar{\mu}(T) \frac{\partial u}{\partial y}\right|_{y=0,1}, q_{w}=-\left.k \frac{\partial T}{\partial y}\right|_{y=0,1}
$$

Substituting equation (14) into (13), we obtain

$$
\begin{aligned}
& \qquad C_{f}=\left.e^{-\varepsilon \theta} \frac{\partial w}{\partial \eta}\right|_{\eta=0,1}, N u=\left.\frac{\partial \theta}{\partial \eta}\right|_{\eta=0,1} \\
& \qquad \operatorname{Re} e^{\varepsilon \theta_{i}} \frac{d w}{d \tau}=\frac{w_{i+1}-2 w_{i}+w_{i-1}}{(\Delta \eta)^{2}}-\varepsilon\left(\frac{\theta_{i+1}-\theta_{i-1}}{2 \Delta \eta}\right) \\
& \text { Pr R e } \frac{d \theta_{i}}{d \tau}=\left(\frac{\theta_{i+1}-2 \theta_{i}+\theta_{i-1}}{(\Delta \eta)^{2}}\right)-\operatorname{Re~Pr}\left(\frac{\theta_{i+1}-\theta_{i-1}}{2 \Delta \eta}\right. \\
& \text { with initial conditions } \\
& \text { where } w_{i}(\tau)=w\left(\eta_{i}, \tau\right), \theta_{i}(\tau)=\theta\left(\eta_{i}, \tau\right), w_{1}=\theta_{1}=0, w_{\mathrm{N}+1} \\
& =\theta_{\mathrm{N}+1}=1 \text { and the spatial interval }[0,1] \text { is partition into } N \\
& \text { equal sub-intervals. The grid size and the grid points are } \\
& \text { define as } \Delta \eta=1 / N \text { and } \eta_{i}=(i-1) \Delta \eta, 1 \leq i \leq N+1 \\
& \text { The resulting nonlinear system of initial value problem is } \\
& \text { then solved iteratively using the initial value solvers like } \\
& \text { Runge-Kutta Fehlberg integration scheme }[11] \text {. }
\end{aligned}
$$

\section{Numerical Procedure (Transient Flow)}

Here, the governing nonlinear equations (8)-(10) are disretized based using finite difference on a linear Cartesian mesh and uniform grid. The second and first spatial derivatives are approximated with second-order central differences. The semi-discretization scheme for the velocity and temperature component reads as:

$$
\begin{array}{r}
\operatorname{Re} e^{\varepsilon \theta_{i}} \frac{d w}{d \tau}=\frac{w_{i+1}-2 w_{i}+w_{i-1}}{(\Delta \eta)^{2}}-\varepsilon\left(\frac{\theta_{i+1}-\theta_{i-1}}{2 \Delta \eta}\right)\left(\frac{w_{i+1}-w_{i-1}}{2 \Delta \eta}\right)-e^{\varepsilon \theta_{i}}\left(\operatorname{Re}\left(\frac{w_{i+1}-w_{i-1}}{2 \Delta \eta}\right)+H a\left(w_{i}-1\right)\right) \\
\operatorname{PrRe} \frac{d \theta_{i}}{d \tau}=\left(\frac{\theta_{i+1}-2 \theta_{i}+\theta_{i-1}}{(\Delta \eta)^{2}}\right)-\operatorname{Re} \operatorname{Pr}\left(\frac{\theta_{i+1}-\theta_{i-1}}{2 \Delta \eta}\right)+E c \operatorname{Pr} e^{-\varepsilon \theta_{i}}\left(\frac{w_{i+1}-w_{i-1}}{2 \Delta \eta}\right)^{2}+\operatorname{EcPr}^{2} \operatorname{Pr}_{\left(w_{i}-1\right)^{2}}
\end{array}
$$

\section{Steady Flow Analysis}

For a given set of parameter values, the reactive third grade flow evolves in time until a steady state condition is attained. Whenever this happens, equations (13)-(17) then become,

$$
\begin{array}{r}
\frac{d^{2} w}{d \eta^{2}}-\varepsilon \frac{d \theta}{d \eta} \frac{d w}{d \eta}-e^{\varepsilon \theta}\left(\operatorname{Re} \frac{d w}{d \eta}+H a(w-1)\right)=0 \\
\frac{d^{2} \theta}{d \eta^{2}}-\operatorname{Re} \operatorname{Pr} \frac{d \theta}{d \eta}+E c \operatorname{Pr} e^{-\varepsilon \theta}\left(\frac{d w}{d \eta}\right)^{2}+E c \operatorname{Pr} H a(w-1)^{2}=0
\end{array}
$$

with

$$
\left.\begin{array}{l}
w(0)=0, \theta(0)=0, \\
w(1)=1, \theta(1)=1,
\end{array}\right\}
$$

It is important to note that $\varepsilon=0$ corresponds to the case of constant viscosity conducting fluid. The exact solution of equation (18) for the fluid velocity is possible under this constant viscosity scenario and we obtain

$$
w(\eta)=1+\frac{e^{a \eta+b}-e^{b \eta+a}}{e^{a}-e^{b}}
$$

where $a=\left(\operatorname{Re}+\sqrt{\operatorname{Re}^{2}+4 H a}\right) / 2$ and $b=\left(\operatorname{Re}-\sqrt{\operatorname{Re}^{2}+4 H a}\right) / 2$. Equations (18)-(20) represent a nonlinear boundary value problem and this precludes its exact solution. In order to tackle the problem numerically, shooting method coupled with Runge-Kutta Fehlberg integration scheme is employed [11]. Firstly, the model nonlinear boundary value problem is reduced to a system of initial value problem. Let

$$
w=x_{1}, w^{\prime}=x_{2}, \theta=x_{3}, \theta^{\prime}=x_{4}
$$

where the prime symbol denotes the derivative with respect to $\eta$. Substituting equation (22) into equations (18)-(20), we obtain

$$
\begin{aligned}
& x_{1}^{\prime}=x_{2}, \\
& x_{2}^{\prime}=\varepsilon x_{2} x_{4}+e^{\varepsilon x_{3}}\left(\operatorname{Re} x_{2}+H a\left(x_{1}-1\right)\right), \\
& x_{3}^{\prime}=x_{4}, \\
& x_{4}^{\prime}=\operatorname{Re} \operatorname{Pr} x_{4}-E c \operatorname{Pr} e^{-\varepsilon x_{3}} x_{2}^{2}-E c \operatorname{Pr}\left(x_{1}-1\right)^{2}
\end{aligned}
$$

subject to the following initial conditions;

$$
x_{1}(0)=0, x_{2}(0)=s_{1}, x_{3}(0)=0, x_{4}(0)=s_{2}
$$

The unspecified initial conditions $s_{1}$ and $s_{2}$ in equation (24) are obtained iteratively using the Newton-Raphson algorithm together with Runge-Kutta Fehlberg integration scheme to a given terminal point $\eta=1$. For a fixed set of parameter values, the accuracy of the missing initial conditions was checked by comparing the calculated value with the given value at the terminal point. The computations were done by a written program in MAPLE with a step size of $\Delta \eta=0.001$ selected to be satisfactory for a convergence criterion of $10^{-7}$ in nearly all cases.

\section{Entropy Analysis}

Magnetohydrodynamic and heat transfer processes in a generalized Couette flows with permeable boundaries are irreversible. This inherent irreversibility arises due to the exchange of energy and momentum within the fluid and at permeable boundaries, thus resulting in entropy generation. Following Wood [2], the local volumetric rate of entropy generation $E_{G}$ for a viscous incompressible conducting 
fluid in the presence of magnetic field is defined by

$$
E_{G}=\frac{k}{T_{0}^{2}}\left(\frac{\partial T}{\partial y}\right)^{2}+\frac{\mu}{T_{0}}\left(\frac{\partial u}{\partial y}\right)^{2}+\frac{\sigma B_{0}^{2}}{T_{0}}(u-U)^{2}
$$

The first term in equation (26) is irreversibility due to heat transfer, the second term is entropy generation due to viscous dissipation and the third term is local entropy generation due to the effect of the Joule dissipation. Substituting equation (8) into equation (25), we obtain the dimensionless form of local entropy generation rate as

$$
N s=\frac{T_{0}^{2} h^{2} E_{G}}{k\left(T_{h}-T_{0}\right)^{2}}=\left(\frac{\partial \theta}{\partial \eta}\right)^{2}+\frac{B r}{\Omega}\left[e^{-\varepsilon \theta}\left(\frac{\partial w}{\partial \eta}\right)^{2}+H a(w-1)^{2}\right]
$$

where $\Omega=\left(T_{h}-T_{0}\right) / T_{0}$ is the temperature difference parameter and $\mathrm{Br}=E c P r$ is the Brinkman number. The Bejan number $\mathrm{Be}$ is define as

$$
B e=\frac{N_{1}}{N_{s}}=\frac{1}{1+\Phi}
$$

where $\mathrm{Ns}=N_{1}+N_{2}$,

$$
\begin{aligned}
& N_{1}=\left(\frac{\partial \theta}{\partial \eta}\right)^{2} \text { (Heat transfer irreversibility), } \\
& N_{2}=\frac{B r}{\Omega}\left[e^{-\varepsilon \theta}\left(\frac{\partial w}{\partial \eta}\right)^{2}+H a(w-1)^{2}\right] \text { (Fluid friction and magnetic }
\end{aligned}
$$

field irreversibility),

$$
\Phi=\frac{N_{2}}{N_{1}} \text { (Irreversibility ratio) }
$$

Equation (27) shows that Bejan number ranges from 0 to 1. $B e=0$ is the limit where the irreversibility is dominated by the combined effects of fluid friction and magnetic fields and $B e=1$ is the limit where the irreversibility due to heat transfer dominates the flow system by virtue of finite temperature differences.

\section{Results and Discussion}

Numerical solution for the representative velocity field, temperature field, skin friction, Nusselt number, entropy generation rate and Bejan number have been carried out by assigning some arbitrary chosen specific values to various thermophysical parameters controlling the flow system (see figures 2 -10). The Prandtl number (Pr) is assigned the values ranging from (Air) $0.71 \leq \operatorname{Pr} \leq 7.1$ (water) which are the most encountered fluids in nature and frequently used in engineering and industries.

\subsection{Transient Velocity and Temperature Profiles}

Figures (2)-(4) illustrated the effects of unsteadiness on the velocity and temperature profiles. Velocity increases from its zero value in time and space at the lower fixed plate to its maximum value at the upper moving plate (see figure 2a,b). Similar trend is observed with temperature profile with lowest value at the lower fixed plate and highest value at the upper moving plate satisfying the prescribed initial and boundary conditions. An increase in time, increases the fluid velocity and temperature across the channel until a steady state is achieved as shown in figures $(3 a, b)$ and figures $(4 a, b)$. Moreover, near the moving upper plate, it is interesting to note that both the velocity and temperature oscillate with decreasing amplitude until a steady state is achieved. The velocity profiles attained the steady state faster than the temperature profiles. For instance, steady state temperature is attained at $\tau \geq 0.6$ while for the same parameter values; steady state velocity is attained at $\tau \geq 0.4$. This may be attributed to the fact that velocity and velocity gradient serve as heat sources for the temperature through Joule heating and viscous dissipation.

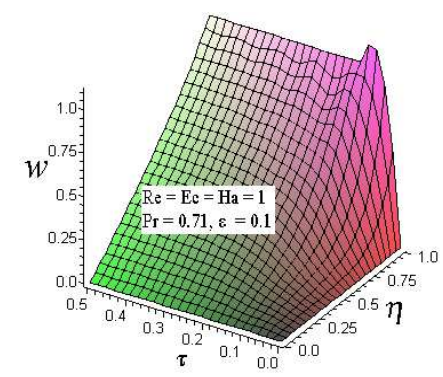

Figure 2a. Fluid velocity profiles across the channel with increasing time

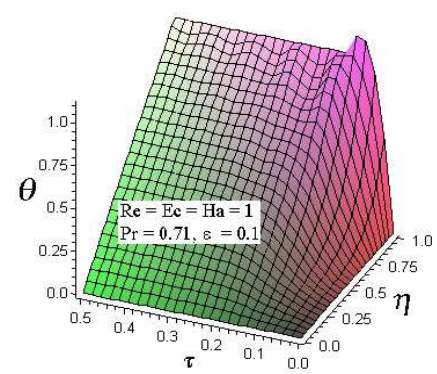

Figure $2 \boldsymbol{b}$. Fluid temperature profiles across the channel with increasing time

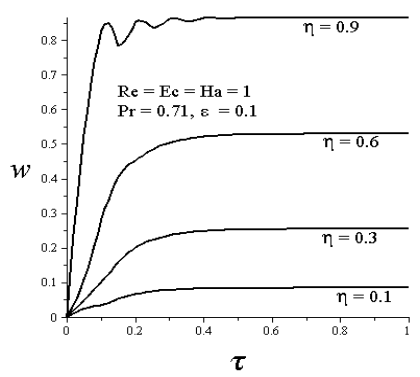

Figure 3a. Fluid velocity profiles across the channel with increasing time

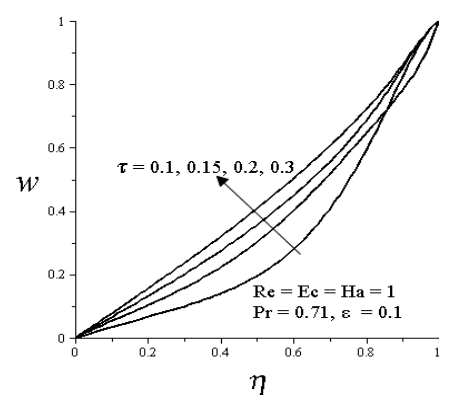

Figure 3b. Fluid velocity profiles across the channel with increasing time 


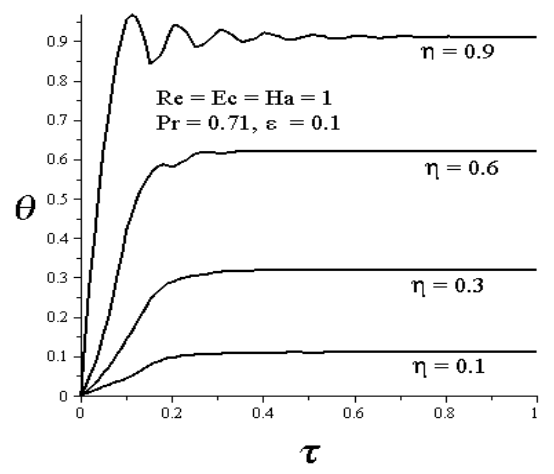

Figure 4a. Fluid temperature profiles across the channel with increasing time

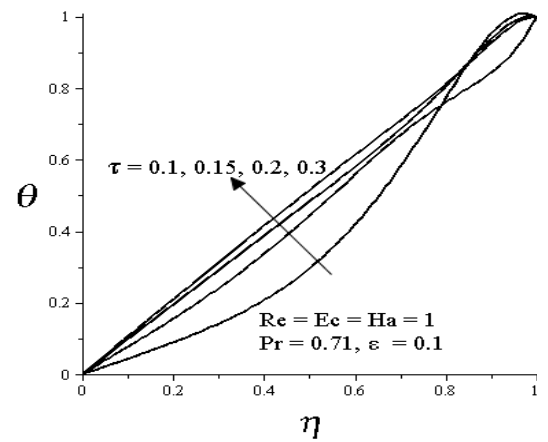

Figure $4 \boldsymbol{b}$. Fluid temperature profiles across the channel with increasing time

\subsection{Steady State Velocity and Temperature Profiles}

The numerical results displayed in table 1 represent a comparison between the exact solution in equation (22) and the shooting method numerical solution. The excellent agreement attests to the credibility of our numerical procedure.

Table 1. Computation showing comparison between the exact and numerical solution of velocity profile for $\mathrm{Re}=1, \mathrm{Ha}=1, \varepsilon=0$.

\begin{tabular}{lll}
\hline $\boldsymbol{\eta}$ & Exact Solution $\boldsymbol{w}(\boldsymbol{\eta})$ & Numerical Solution $\boldsymbol{w}(\boldsymbol{\eta})$ \\
\hline 0 & 0 & 0 \\
0.1 & 0.08812141 & 0.08812141 \\
0.2 & 0.17591234 & 0.17591234 \\
0.3 & 0.26426209 & 0.26426209 \\
0.4 & 0.35415939 & 0.35415939 \\
0.5 & 0.44671325 & 0.44671325 \\
0.6 & 0.54317727 & 0.54317727 \\
0.7 & 0.64497804 & 0.64497804 \\
0.8 & 0.75374838 & 0.75374838 \\
0.9 & 0.87136619 & 0.87136619 \\
1.0 & 1 & 1 \\
\hline
\end{tabular}

Figures $(5 \mathrm{a}, \mathrm{b})$ illustrate the effects of increasing parameters $\mathrm{Ha}, \mathrm{Re}$ and $\varepsilon$ on steady state velocity profiles. An increase in magnetic field intensity (i.e. Ha increasing values) causes a decline in the fluid motion towards the upper moving plate. This may be attributed to the resistive force called Lorentz force which tends to oppose the fluid motion toward the upper plate. The trend is opposite with increasing $R e$, leading to a rise in the fluid motion towards the upper moving plate due to increasing suction. An increase in the viscosity exponent $\mathcal{E}$ causes a rise in the fluid motion towards the upper moving plate as a result of decreasing fluid viscosity.

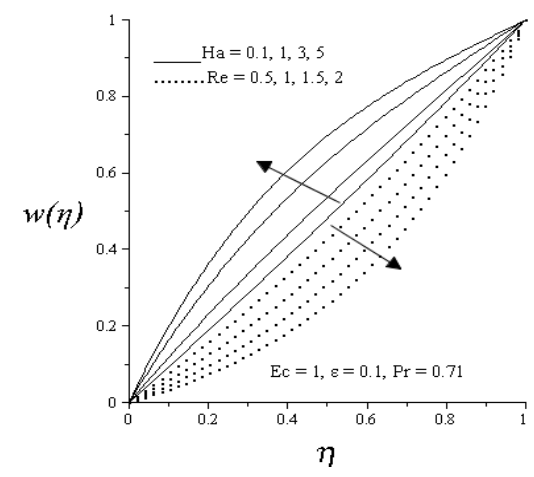

Figure 5a. Effect of increasing Ha and Re on velocity profiles

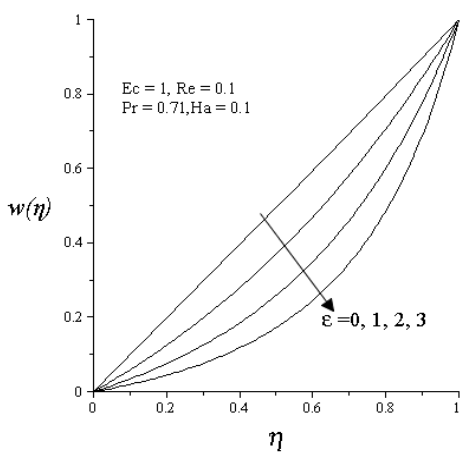

Figure 5 b. Effect of increasing $\varepsilon$ on velocity profiles

Figures $(6 a, b)$ depict the effects of increasing Ha, Re, $\mathrm{Pr}$ and Ec on temperature profiles. A rise in magnetic field intensity (Ha) causes an increase in the fluid temperature within he channel. This may be explained as the effect of internal heating generation due to Joule dissipation. An increase in $R e$ due to the combined effects of fluid injection/suction causes a fall in the fluid temperature. As in $E c$ and $P r$ rise, the fluid temperature increases due to viscous heating effect, this acts as a source to temperature field. It is noteworthy that elevation in temperature field is more pronounced at high Prandtl number $(\mathrm{Pr}=7.1)$ in comparison to that of low Prandtl number $(\operatorname{Pr}=0.71)$ flow.

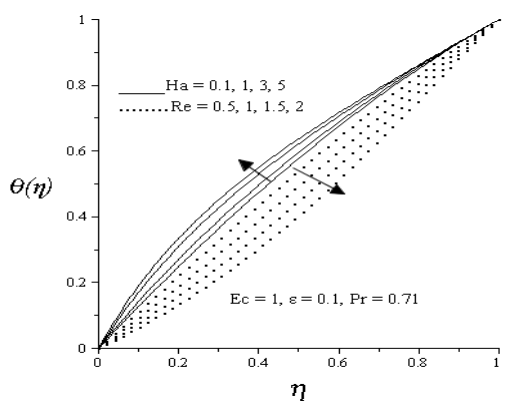

Figure 6a. Effect of increasing Ha, Re on temperature profiles 


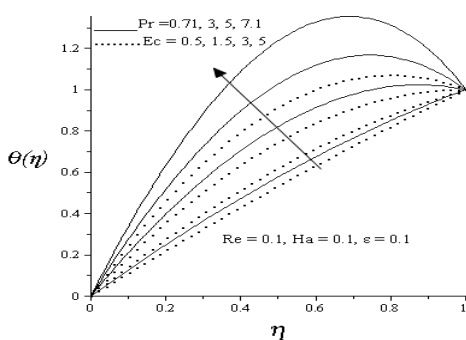

Figure 6b. Effect of increasing $P r$, Ec on temperature profiles

\subsection{Skin Friction and Nusselt Number}

Figures (7) -(8) illustrate the effects of increasing Ha, Re, $\varepsilon$ on the skin friction and the rate of heat transfer at both lower fixed plate and the upper moving plate. The skin friction reduces with an increase in $R e$ and $\varepsilon$ but increases with a rise in $H a$ at the lower fixed plate. The trend is reversed at the upper moving plate. The skin friction decreases with an elevation in the magnetic field intensity but increases with an increase in Re and $\varepsilon$ (see figures 7a,b). This may be attributed to the fact the a decrease in fluid viscosity enhances the velocity gradient at the upper moving plate while the Lorentz force due to magnetic field acts in opposite manner. In figure $(8 \mathrm{a}, \mathrm{b})$, the Nusselt number $N u$ decreases with an increase in $R e$ and $\varepsilon$ but increases with an increase in $\mathrm{Ha}$ at the lower fixed plate surface. Meanwhile, the rate of heat transfer at the upper moving plate increases with an increase in Re, $\varepsilon$ and $H a$. This is expected, since temperature gradient at the upper moving plate increases due to a rise in fluid suction and a decline in fluid viscosity, consequently, the heat transfer rate increases. Increases in $\mathrm{Ha}$ in the presence of suction also enhance the temperature gradient slightly at the upper moving plate.

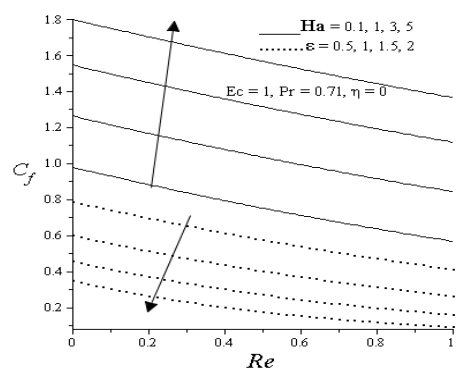

Figure 7a. Skin friction at the lower plate with increasing $\mathrm{Ha}, \mathrm{Re}, \boldsymbol{\varepsilon}$.

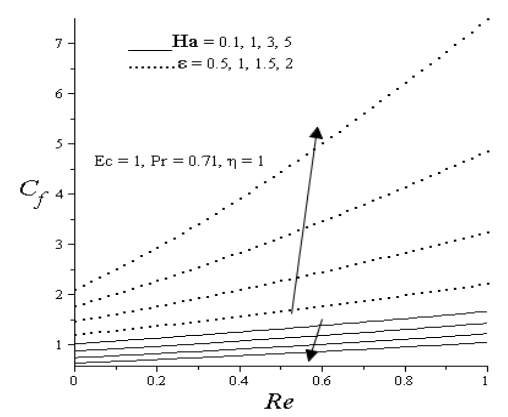

Figure 7b. Skin friction at the upper plate with increasing $\mathrm{Ha}, \mathrm{Re}, \boldsymbol{\varepsilon}$.

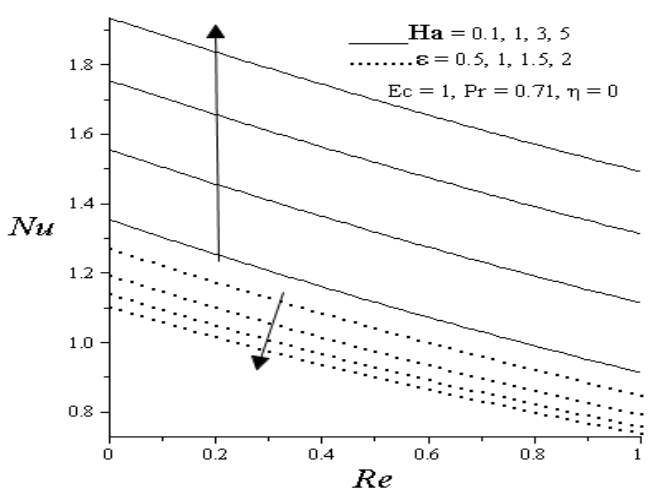

Figure 8a. Nusselt number at the lower plate with increasing $\mathrm{Ha}, \mathrm{Re}, \mathrm{\varepsilon}$.

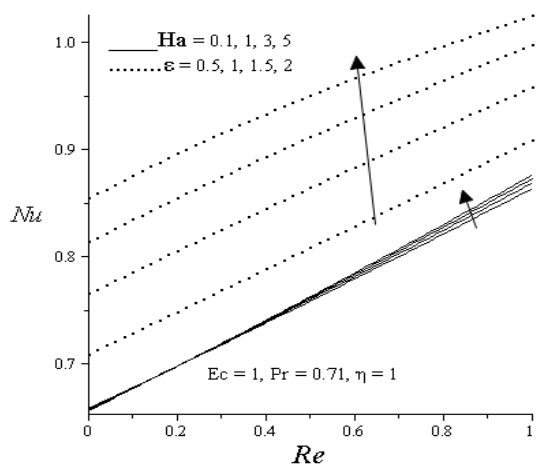

Figure 8b. Nusselt number at the upper plate with increasing $\mathrm{Ha}$, Re, $\varepsilon$.

\subsection{Entropy Generation Rate}

Figures (9a,b) illustrate the effects of increasing $\mathrm{Ha}, \mathrm{Re}$, $\operatorname{Br} \Omega^{-1}$ and $\varepsilon$ on local entropy generation rate. The presence of the magnetic field $(\mathrm{Ha})$ causes a decline in the entropy generation across the channel with more entropy generation at the lower fixed plate and less entropy production at the upper moving plate. A reverse trend is observed with an increase in Re. The $N s$ is depressed at the lower fixed plate and enhanced at the upper moving plate with increasing $R e$. An increase in group parameter $\mathrm{Br} \Omega^{-1}$ due to viscous dissipation causes the entropy production to increase. Less entropy is generated at the lower fixed plate and more at the upper moving plate with increasing viscosity exponent $\varepsilon$ due to a decrease in fluid viscosity.

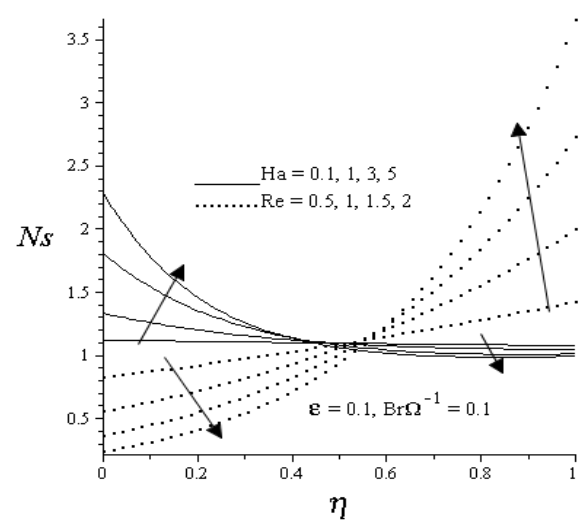

Figure 9a. Effect of increasing Ha and Re on entropy generation rate. 


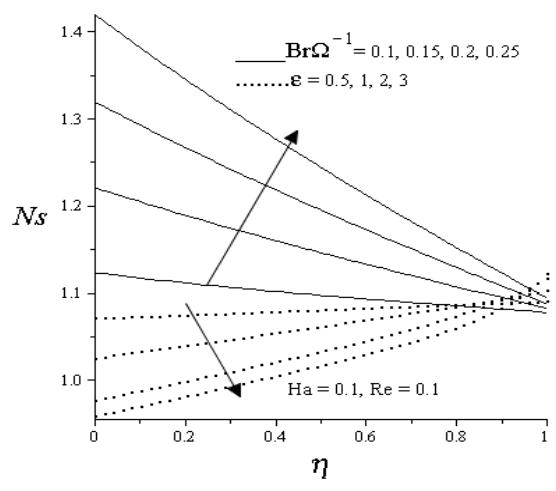

Figure 9b. Effect of increasing $B r \Omega^{I}$ and $\varepsilon$ on entropy generation rate

\subsection{Bejan Number}

Figures $(10 \mathrm{a}, \mathrm{b})$ depict the effects of increasing $\mathrm{Ha}, \mathrm{Re}$, $B r \Omega^{-1}$ and $\varepsilon$ on Bejan number. Irreversibility due to fluid friction and Joule heating become dominant at the lower fixed plate region while the heat transfer irreversibility become dominant at the upper moving plate region with increasing magnetic field (Ha). Increasing $R e$ causes heat transfer irreversibility to intensify at the lower fixed plate while viscous and Joule heating irreversibility becomes dominant at the upper moving plate. The group parameter $\mathrm{Br} \Omega^{-1}$ increases the fluid friction and Joule heating irreversibility across the channel. Moreover, a decrease in fluid viscosity increases the dominant effect of heat transfer irreversibility.

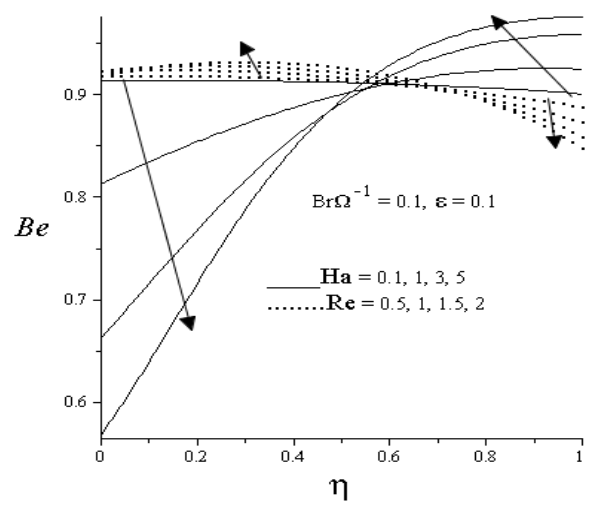

Figure 10a. Effect of increasing Ha and Re on Bejan number.

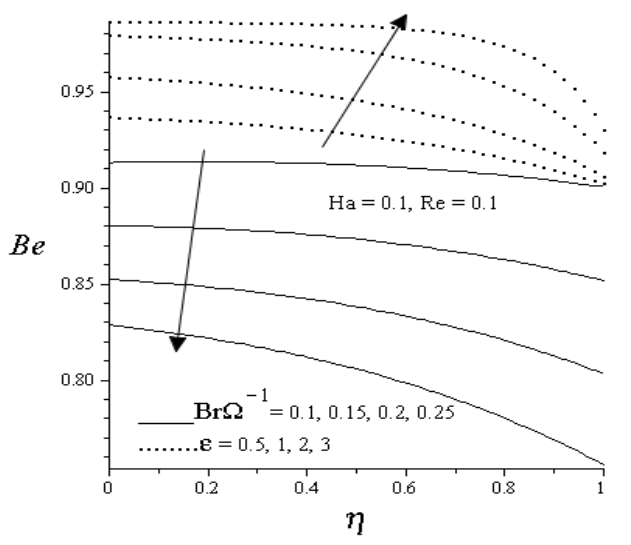

Figure 10b. Effect of increasing $B r \Omega^{l}$ and $\mathcal{E}$ on Bejan number.

\section{Conclusions}

Computational model and thermodynamic analysis of a temperature dependent viscosity hydromagnetic generalized unsteady Couette flow with permeable walls is presented. Using a semi-discretization method together with shooting iteration scheme both the transient and steady state problem are numerically tackled. Some of the results obtained can be summarized as follows:

- Both the fluid velocity and temperature increases with time across the channel until a steady state is achieved. The velocity profiles attained steady state faster than the temperature profiles.

- Increase in $\mathrm{Re}, \varepsilon$ increases fluid motion towards the upper moving plate while increase in $\mathrm{Ha}$ increases fluid motion towards the lower fixed plate.

- Fluid temperature at the lower fixed plate region increases with $\mathrm{Ha}, \mathrm{Ec}, \operatorname{Pr}$ while increase in $\mathrm{Re}, \varepsilon$ increases the temperature at the upper moving plate region.

- $\quad$ Skin friction increases with $\mathrm{Ha}$ but decreases with $\mathrm{Re}$ and $\mathcal{E}$ at the lower fixed plate. The situation is reversed at the upper moving plate.

- $\quad$ Nusselt number increases with $H a, R e, \varepsilon$ at the upper moving plate while at the lower fixed plate, $\mathrm{Nu}$ decreases with $\mathrm{Re}, \varepsilon$, and increases with $H a$.

- Local entropy generation rate increases with $\mathrm{Br} \Omega^{-1}$ but decreases with $\varepsilon$. Increase in $\mathrm{Ha}$ decreases entropy production at the moving upper plate while increase in Re decreases entropy generation at the lower fixed plate.

- A decrease in fluid viscosity increases Bejan number while an increase group parameter $\mathrm{Br} \Omega^{-1}$ decreases Bejan number. Increase in $R e$ increases $B e$ at the lower fixed plate and decreases $B e$ at the upper moving plate. The situation is reversed with increasing $\mathrm{Ha}$.

\section{References}

[1] Paoletti, S., Rispoli, F., Sciubba, E. (1980), Calculation of exergetic losses in compact heat exchanger passages, ASME AES, 10, pp. 21-29.

[2] Wood L.C. (1975), Thermodynamics of Fluid Systems, Oxford University Press, Oxford, (1975).

[3] Bejan, A. (1982), Entropy Generation through heat and fluid flow, chapter, 2, New York, USA.

[4] Bejan, A. (1988), Advanced Engineering Thermodynamics, Wiley, USA.

[5] Jery, A.E., Hidouri, N., Magherbi, M., Brahim, A.B. (2010), Effect of an external oriented magnetic field on entropy generation in natural convection, Entropy, 12, pp. 13911417.

[6] Salas, H., Cuevas, S., Haro, M.L. (1999), Entropy generation analysis of magnetohydrodynamic induction devices, J. Phys D: Appl Phys. 32, pp.2605-2608. 
[7] Mahmud, S., Fraser, R.A. (2004), Magnetohydrodynamic free convection and entropy generation in a square porous cavity, Int. J. Heat Mass Transfer, 47, pp. 3245-3256.

[8] Ibanez, G., Cuevas, S. (2008), Optimum wall conductance ratio in magnetoconvective flow in a long vertical rectangular duct, Int. J. Thermal Sci., 47, pp. 1012-1019.

[9] Eegunjobi A.S., Makinde O.D. (2012), Effects of Navier slip on entropy generation in a porous channel with suction/injection. Journal of Thermal Science and
Technology, 7(4), $522-535$.

[10] Makinde O.D., Eegunjobi A.S. (2013), Effects of convective heating on entropy generation rate in a channel with permeable walls. Entropy 15, 220-233.

[11] Na T.Y. (1979), Computational methods in engineering boundary value problems, Academic press, New York. 